\title{
Periodic Solutions and Nontrivial Periodic Solutions for a Class of Rayleigh-Type Equation with Two Deviating Arguments
}

\author{
Meiqiang Feng \\ School of Applied Science, Beijing Information Science \& Technology University, Beijing 100192, China \\ Correspondence should be addressed to Meiqiang Feng; meiqiangfeng@sina.com
}

Received 27 May 2013; Accepted 27 August 2013

Academic Editor: Henryk Hudzik

Copyright (C) 2013 Meiqiang Feng. This is an open access article distributed under the Creative Commons Attribution License, which permits unrestricted use, distribution, and reproduction in any medium, provided the original work is properly cited.

The Rayleigh equation with two deviating arguments $x^{\prime \prime}(t)+f\left(x^{\prime}(t)\right)+g_{1}\left(t, x\left(t-\tau_{1}(t)\right)\right)+g_{2}\left(t, x\left(t-\tau_{2}(t)\right)\right)=e(t)$ is studied. By using Leray-Schauder index theorem and Leray-Schauder fixed point theorem, we obtain some new results on the existence of periodic solutions, especially for the existence of nontrivial periodic solutions to this equation. The results are illustrated with two examples, which cannot be handled using the existing results.

\section{Introduction}

Consider the Rayleigh equation with two deviating arguments in the form of

$$
\begin{aligned}
x^{\prime \prime} & (t)+f\left(x^{\prime}(t)\right)+g_{1}\left(t, x\left(t-\tau_{1}(t)\right)\right)+g_{2}\left(t, x\left(t-\tau_{2}(t)\right)\right) \\
& =e(t),
\end{aligned}
$$

where

$$
\begin{gathered}
f \in C(R, R), \quad g_{i} \in C(R \times R, R), \quad i=1,2, \\
e, \tau_{i} \in C(R, R), \quad i=1,2, \\
g_{i}(t+T, x)=g_{i}(t, x), \quad \tau_{i}(t+T)=\tau_{i}(t), \quad i=1,2, \\
e(t+T)=e(t) .
\end{gathered}
$$

The dynamic behaviors of Rayleigh equation have been widely investigated due to their applications in many fields such as physics, mechanics, and the engineering technique fields. For example, an excess voltage of ferroresonance, known as some kind of nonlinear resonance having long duration that arises from the magnetic saturation of inductance in an oscillating circuit of a power system, and a boosted excess voltage can give rise to some problems in relay protection. To probe this mechanism, a mathematical model was proposed in [1-3], which is a special case of the Rayleigh equation with two delays. This implies that (1) can represent analog voltage transmission. In a mechanical problem, $f$ usually represents a damping or friction term, $g_{i}$ represents the restoring force, $e$ is an externally applied force, and $\tau_{i}$ is the time lag of the restoring force (see [4]). Some other examples in practical problems concerning physics and engineering technique fields can be found in [5-7].

At the same time, the periodic solutions for Rayleigh equations with two deviating arguments have been studied by authors [8-10] under the assumption of

$$
f(0)=0 \quad \text { or } \quad f(t, 0)=0 .
$$

It is not difficult to see that if $g_{1}(t, 0)+g_{2}(t, 0) \quad \neq \equiv(t)$, then the periodic solution obtained in [8-10] must be nontrivial. But if $g_{1}(t, 0)+g_{2}(t, 0) \equiv e(t)$, then the periodic solution obtained in [8-10] may be trivial under the assumption of $f(0)=0$ or $f(t, 0)=0$. And if the periodic solution is unique, then it must be trivial. Thus, it is worth discussing the existence of the nontrivial periodic solutions of Rayleigh equations with two deviating arguments in this case.

The main purpose of this paper is to establish sufficient conditions for the existence of periodic solution, especially for the existence of nontrivial periodic solutions of (1) by using the Leray-Schauder index theory. We remark that our methods are different from those used in [8-10] to some degree. In particular, two examples are also given to illustrate the effectiveness of our results. 
For ease of exposition, throughout this paper, we assume that $T>0$.

On the other hand, the following assumptions are used in this paper.

$\left(H_{1}\right) f \in C(R, R), g_{i} \in C\left(R^{2}, R\right), \tau_{i} \in C(R, R), i=1,2, e \in$ $C(R, R), g_{i}(t+T, x)=g_{i}(t, x), \tau_{i}(t+T)=\tau_{i}(t), i=$ 1,2 , and $e(t+T)=e(t)$.

$\left(H_{2}\right) f(0)=0$, and there exists $\gamma>0$ such that $x f(x) \geq$ $\gamma|x|^{2}$, for all $x \in R$ (or $x f(x) \leq-\gamma|x|^{2}$, for all $\left.x \in R\right)$.

$\left(H_{3}\right) g_{i}$ is differentiable with respect to $t$, and there exist $a_{i} \geq 0, b_{i}>0, i=1,2$, such that

$$
\left|g_{i t}^{\prime}(t, x)\right| \leq a_{i}+b_{i}|x|, \quad \forall(t, x) \in R^{2}, i=1,2 .
$$

$\left(H_{4}\right)$ There exist $l_{i}>0$ such that $\left|g_{i}\left(t, x_{1}\right)-g_{i}\left(t, x_{2}\right)\right| \leq$ $l_{i}\left|x_{1}-x_{2}\right|$, for all $t \in R, x_{1}, x_{2} \in R, i=1,2$.

$\left(H_{5}\right)$ There exists $d>0$, such that for $|x|>d$, we have

$$
\left(g_{1}(t, x)+g_{2}(t, x)-e(t)\right) \operatorname{sign} x>0, \quad \forall t \in R .
$$

$\left(H_{6}\right)$ There exist integers $n_{i}$ such that $\delta_{i} \triangleq \max _{t \in[0, T]} \mid \tau_{i}(t)-$ $n_{i} T \mid \leq T, i=1,2$.

The main results of the present paper are as follows.

Theorem 1. If $\left(H_{1}\right)-\left(H_{6}\right)$ hold, and $\left(b_{1}+b_{2}\right) \gamma^{-1} T^{2}+2 \sqrt{2}\left(l_{1} \delta_{1}+\right.$ $\left.l_{2} \delta_{2}\right) \pi^{2} \gamma^{-1}<2 \pi^{2}$, then (1) has at least one periodic solution.

The proof of Theorem 1 will be given in Section 3 .

Remark 2. If $g_{1}(t, 0)+g_{2}(t, 0) \not \equiv e(t)$, then the periodic solution obtained by Theorem 1 must be nontrivial. If $g_{1}(t, 0)+$ $g_{2}(t, 0) \equiv e(t)$, we could not conclude whether or not the periodic solution is nontrivial.

So we give the following conditions:

$\left(H_{7}\right) g_{i x}^{\prime}(t, x), i=1,2$ exist and are continuous, $g_{1 x}^{\prime}(t, 0)+$
$g_{2 x}^{\prime}(t, 0) \equiv$ const. $<0$. $\left(H_{8}\right) \lim _{x \rightarrow 0}(f(x) / x)=r \geq \gamma$.

Theorem 3. If $\left(H_{1}\right)-\left(H_{8}\right)$ hold, and $g_{1}(t, 0)+g_{2}(t, 0) \equiv$ $e(t),\left(b_{1}+b_{2}\right) \gamma^{-1} T^{2}+2 \sqrt{2}\left(l_{1} \delta_{1}+l_{2} \delta_{2}\right) \pi^{2} \gamma^{-1}<2 \pi^{2}$, then (1) has at least one nontrivial periodic solution.

The proof of Theorem 3 will be given in Section 4 .

\section{Preliminaries}

In this section, to establish the periodic solutions of (1), we provide some background definitions and some well-known results, which are crucial in our arguments.

Let $X$ be a real Banach space, and let $A: X \rightarrow X$ be a completely continuous operator.

Definition 4 (see $[11,12]$ ). If $x_{0}$ is an isolated fixed point of $A$, then the fixed point index at $x_{0}$ of $A$ is defined by

$$
i\left(A, x_{0}\right)=\operatorname{deg}(I-A, \Omega, \theta)
$$

where $\Omega$ is a neighborhood of $x_{0}$, which satisfies that $x_{0}$ is the unique fixed point in $\Omega$ of $A$.

Definition 5 (see $[11,12]$ ). If there exits $x_{0} \in X$ with $x_{0} \neq \theta$ such that $A x_{0}=\lambda x_{0}, \lambda \in R$, then $\lambda$ is called an eigenvalue of operator $A$ and $x_{0}$ is called the eigenfunction of operator $A$ corresponding to $\lambda$.

Definition 6. Let $u: R \rightarrow R$ be continuous. $u(t)$ is said to be periodic on $R$ if

$$
u(t+T)=u(t), \quad \forall t \in R .
$$

Lemma 7 (see [11, 12], index theorem of Leray-Schauder). Suppose that $x_{0}$ is a fixed point of $A, A$ is Fréchet differentiable at $x_{0}$, and 1 is not the eigenvalue of $A^{\prime}\left(x_{0}\right)$. Then $x_{0}$ is an isolated fixed point of $A$, and

$$
i\left(A, x_{0}\right)=i\left(A^{\prime}\left(x_{0}\right), 0\right)=(-1)^{\beta},
$$

where $\beta$ is equal to the sum of the algebraic multiplicities of all of the eigenvalues $\lambda_{i}>1$ of $A^{\prime}\left(x_{0}\right)$.

Lemma 8 (see $[11,12]$, fixed point theorem of Leray-Schauder). Let $X$ be a real Banach space and $A: X \rightarrow X$ be a completely continuous operator. If

$$
\{x: x \in X, x=\lambda A x, 0<\lambda<1\}
$$

is bounded, then $A$ has a fixed point $x^{*} \in \Omega$, where

$$
\begin{gathered}
\Omega=\{x: x \in X,\|x\| \leq l\}, \\
l=\sup \{x: x \in X, x=\lambda A x, 0<\lambda<1\} .
\end{gathered}
$$

Lemma 9. Suppose $x(t) \in C^{1}[0, T]$ and $x(0)=x(T)=0$. Then $\int_{0}^{T}|x(t)|^{2} d t \leq\left(T^{2} / \pi^{2}\right) \int_{0}^{T}\left|x^{\prime}(t)\right|^{2} d t$.

Lemma 10 (see [13]). Let $0 \leq \alpha \leq T$ be constant, $s \in C(R, R)$ be periodic with period $T$, and $\max _{t \in[0, T]}|s(t)| \leq \alpha$. Then for any $u \in C^{1}(R, R)$ which is periodic with period $T$, we have

$$
\int_{0}^{T}|u(t)-u(t-s(t))|^{2} d t \leq 2 \alpha^{2} \int_{0}^{T}\left|u^{\prime}(t)\right|^{2} d t .
$$

\section{Proof of Theorem 1}

In this section, we will use Lemma 8 to prove Theorem 1.

Proof. Let

$$
\begin{aligned}
& X=\{x: x \in C(R, R), x(t+T)=x(t)\}, \\
& Y=\left\{x: x \in C^{1}(R, R), x(t+T)=x(t)\right\} .
\end{aligned}
$$

Then $X$ and $Y$ are real Banach spaces endowed with the norm

$$
\begin{aligned}
& \|x\|_{\infty}=\max _{t \in[0, T]}|x(t)|, \\
& \|x\|=\|x\|_{\infty}+\left\|x^{\prime}\right\|_{\infty},
\end{aligned}
$$

respectively. 
Choosing $m>0$ with $m \neq(2 k \pi / T)^{2}(k=1,2, \ldots)$, then

$$
x^{\prime \prime}(t)+m x(t)=0
$$

has only trivial solution in $Y$.

In fact, it is easy to see that the general solution of $x^{\prime \prime}(t)+$ $m x(t)=0$ is

$$
x(t)=c_{1} \sin \sqrt{m} t+c_{2} \cos \sqrt{m} t
$$

By the periodic boundary conditions we obtain that $x=0$ is its unique solution in $Y$. Then for $h \in X$,

$$
-x^{\prime \prime}(t)-m x(t)=h(t)
$$

has unique solution $x \in Y$. Writing $x=K h$, then $K: X \rightarrow Y$ is a completely continuous operator.

Define an operator $G: Y \rightarrow X$ by

$$
\begin{aligned}
(G x)(t)= & f\left(x^{\prime}(t)\right)+g_{1}\left(t, x\left(t-\tau_{1}(t)\right)\right) \\
& +g_{2}\left(t, x\left(t-\tau_{2}(t)\right)\right)-m x(t)-e(t), \quad x \in Y .
\end{aligned}
$$

Then $G: Y \rightarrow X$ is continuous and bounded. Let $A=K G: Y \rightarrow Y$. Then $A$ is also a completely continuous operator. By Lemma 8, if

$$
\{x: x \in Y, x=\lambda A x, 0<\lambda<1\}
$$

is bounded in $Y$, then $A$ has a fixed point in $Y$. Thus, (1) has periodic solution.

Now suppose that $x \in Y, 0<\lambda<1$ satisfying $x=\lambda A x$. Then $x(t)$ is a solution of

$$
\begin{aligned}
x^{\prime \prime}(t) & +\lambda f\left(x^{\prime}(t)\right)+\lambda g_{1}\left(t, x\left(t-\tau_{1}(t)\right)\right) \\
& +\lambda g_{2}\left(t, x\left(t-\tau_{2}(t)\right)\right)+(1-\lambda) m x(t) \\
= & \lambda e(t) .
\end{aligned}
$$

Let $t, \bar{t}$ be the maximum point and minimum point of $x(t)$ on $[0, T]$, respectively. Then

$$
x^{\prime}(\underline{t})=0, \quad x^{\prime \prime}(\underline{t}) \leq 0, \quad x^{\prime}(\bar{t})=0, \quad x^{\prime \prime}(\bar{t}) \geq 0 .
$$

Noticing $f(0)=0$, we have

$$
\begin{aligned}
& \lambda g_{1}\left(\underline{t}, x\left(\underline{t}-\tau_{1}(\underline{t})\right)\right)+\lambda g_{2}\left(\underline{t}, x\left(\underline{t}-\tau_{2}(\underline{t})\right)\right) \\
& +(1-\lambda) m x(\underline{t})-\lambda e(\underline{t}) \geq 0, \\
& \lambda g_{1}\left(\bar{t}, x\left(\bar{t}-\tau_{1}(\bar{t})\right)\right)+\lambda g_{2}\left(\bar{t}, x\left(\bar{t}-\tau_{2}(\bar{t})\right)\right) \\
& \quad+(1-\lambda) m x(\bar{t})-\lambda e(\bar{t}) \leq 0,
\end{aligned}
$$

and hence, there exists $\xi \in[\underline{t}, \bar{t}]$ (or $[\bar{t}, \underline{t}])$ such that

$$
\begin{gathered}
\lambda g_{1}\left(\xi, x\left(\xi-\tau_{1}(\xi)\right)\right)+\lambda g_{2}\left(\xi, x\left(\xi-\tau_{2}(\xi)\right)\right) \\
-\lambda e(\xi)+(1-\lambda) m x(\xi)=0,
\end{gathered}
$$

which implies that

$$
\begin{aligned}
\lambda & {\left[g_{1}\left(\xi, x\left(\xi-\tau_{1}(\xi)\right)\right)+g_{2}\left(\xi, x\left(\xi-\tau_{2}(\xi)\right)\right)-e(\xi)\right] } \\
& \times \operatorname{sign} x(\xi)+(1-\lambda) m|x(\xi)|=0 .
\end{aligned}
$$

From $\left(H_{5}\right)$ we know that $|x(\xi)| \leq d$ (see [10]). So we have

$$
|x(t)|=\left|x(\xi)+\int_{\xi}^{t} x^{\prime}(s) d s\right| \leq d+\sqrt{T}\left\|x^{\prime}\right\|_{L^{2}},
$$

and then,

$$
\|x\|_{\infty} \leq d+\sqrt{T}\left\|x^{\prime}\right\|_{L^{2}}
$$

where $\|\cdot\|_{L^{2}}$ is the norm of $L^{2}[0, T]$.

Multiplying (19) with $x^{\prime}(t)$ and integrating from 0 to $T$, then we have

$$
\begin{aligned}
\lambda \int_{0}^{T} f\left(x^{\prime}(t)\right) x^{\prime}(t) d t= & -\lambda \int_{0}^{T} g_{1}\left(t, x\left(t-\tau_{1}(t)\right)\right) x^{\prime}(t) d t \\
& +\lambda \int_{0}^{T} g_{2}\left(t, x\left(t-\tau_{2}(t)\right)\right) x^{\prime}(t) d t \\
& +\lambda \int_{0}^{T} e(t) x^{\prime}(t) d t .
\end{aligned}
$$

By $\left(H_{2}\right)$ we know that

$$
\int_{0}^{T} f\left(x^{\prime}(t)\right) x^{\prime}(t) d t \geq \gamma \int_{0}^{T}\left|x^{\prime}(t)\right|^{2} d t .
$$

By Hölder's inequality, from (26) and (27) we have

$$
\begin{aligned}
& \gamma \int_{0}^{T} x^{\prime 2}(t) d t \\
& \leq\left|\int_{0}^{T} g_{1}\left(t, x\left(t-\tau_{1}(t)\right)\right) x^{\prime}(t) d t\right| \\
& +\left|\int_{0}^{T} g_{2}\left(t, x\left(t-\tau_{2}(t)\right)\right) x^{\prime}(t) d t\right|+\|e\|_{L^{2}}\left\|x^{\prime}\right\|_{L^{2}} \\
& \leq \int_{0}^{T}\left|g_{1}\left(t, x\left(t-\tau_{1}(t)\right)\right)-g_{1}(t, x(t))\right|\left|x^{\prime}(t)\right| d t \\
& +\left|\int_{0}^{T} g_{1}(t, x(t)) x^{\prime}(t) d t\right| \\
& \quad+\int_{0}^{T}\left|g_{2}\left(t, x\left(t-\tau_{2}(t)\right)\right)-g_{2}(t, x(t))\right|\left|x^{\prime}(t)\right| d t \\
& \quad+\left|\int_{0}^{T} g_{2}(t, x(t)) x^{\prime}(t) d t\right|+\|e\|_{L^{2}}\left\|x^{\prime}\right\| L_{L^{2}} \cdot
\end{aligned}
$$

Since $\int_{0}^{x(t)} g_{i}(t, v) d v, i=1,2$ are $T$-periodic, differentiable, and

$$
\begin{aligned}
\frac{d}{d t} \int_{0}^{x(t)} g_{i}(t, v) d v= & g_{i}(t, x(t)) x^{\prime}(t) \\
& +\int_{0}^{x(t)} g_{i t}^{\prime}(t, v) d v, \quad i=1,2,
\end{aligned}
$$


we have

$$
\begin{array}{r}
\int_{0}^{T} g_{i}(t, x(t)) x^{\prime}(t) d t=-\int_{0}^{T} d t \int_{0}^{x(t)} g_{i t}^{\prime}(t, v) d v \\
i=1,2 .
\end{array}
$$

Combining (28) and (30) with $\left(H_{3}\right)$ and $\left(H_{4}\right)$ we obtain

$$
\begin{aligned}
& \gamma \int_{0}^{T} x^{\prime 2}(t) d t \\
& \leq l_{1} \int_{0}^{T}\left|x(t)-x\left(t-\tau_{1}(t)\right)\right|\left|x^{\prime}(t)\right| d t \\
& \quad+l_{2} \int_{0}^{T}\left|x(t)-x\left(t-\tau_{2}(t)\right)\right|\left|x^{\prime}(t)\right| d t \\
& +\int_{0}^{T} d t \int_{0}^{|x(t)|}\left(a_{1}+b_{1}|v|\right) d v \\
& \quad+\int_{0}^{T} d t \int_{0}^{|x(t)|}\left(a_{2}+b_{2}|v|\right) d v+\|e\|_{L^{2}}\left\|x^{\prime}\right\|_{L^{2}} \\
& \leq l_{1}\left\|x^{\prime}\right\|_{L^{2}}\left(\int_{0}^{T}\left|x(t)-x\left(t-\tau_{1}(t)-n_{1} T\right)\right|^{2} d t\right)^{1 / 2} \\
& \quad+l_{2}\left\|x^{\prime}\right\|_{L^{2}}\left(\int_{0}^{T}\left|x(t)-x\left(t-\tau_{2}(t)-n_{2} T\right)\right|^{2} d t\right)^{1 / 2} \\
& \quad+\left(a_{1}+a_{2}\right) \int_{0}^{T}|x(t)| d t+\frac{b_{1}+b_{2}}{2} \int_{0}^{T}|x(t)|^{2} d t \\
& +\|e\|_{L^{2}}\left\|x^{\prime}\right\|_{L^{2}} \cdot
\end{aligned}
$$

By Lemma 9 we have

$$
\begin{aligned}
\int_{0}^{T}|x(t)|^{2} d t= & \int_{\xi}^{\xi+T}|x(t)|^{2} d t=\int_{\xi}^{\xi+T}|x(t)-x(\xi)|^{2} d t \\
& +2 x(\xi) \int_{\xi}^{\xi+T} x(t) d t-|x(\xi)|^{2} T \\
\leq & \frac{T^{2}}{\pi^{2}} \int_{\xi}^{\xi+T}\left|x^{\prime}(t)\right|^{2} d t+2 d \int_{0}^{T}|x(t)| d t \\
& -|x(\xi)|^{2} T \\
\leq & \frac{T^{2}}{\pi^{2}} \int_{0}^{T}\left|x^{\prime}(t)\right|^{2} d t+2 d \int_{0}^{T}|x(t)| d t .
\end{aligned}
$$

By (32) and Hölder's inequality we have

$$
\begin{aligned}
& \int_{0}^{T}|x(t)| d t \\
& \leq \sqrt{T}\left(\int_{0}^{T}|x(t)|^{2} d t\right)^{1 / 2} \\
& \leq \sqrt{T}\left(\frac{T^{2}}{\pi^{2}} \int_{0}^{T}\left|x^{\prime}(t)\right|^{2} d t+2 d \int_{0}^{T}|x(t)| d t\right)^{1 / 2} \\
& \leq \frac{T^{3 / 2}}{\pi}\left(\int_{0}^{T}\left|x^{\prime}(t)\right|^{2} d t\right)^{1 / 2}+\sqrt{2 d T}\left(\int_{0}^{T}|x(t)| d t\right)^{1 / 2} \\
& \leq \frac{T^{3 / 2}}{\pi}\left(\int_{0}^{T}\left|x^{\prime}(t)\right|^{2} d t\right)^{1 / 2}+\frac{1}{4} \int_{0}^{T}|x(t)| d t+2 d T .
\end{aligned}
$$

From (33) we have

$$
\int_{0}^{T}|x(t)| d t \leq \frac{4 T^{3 / 2}}{3 \pi}\left(\int_{0}^{T}\left|x^{\prime}(t)\right|^{2} d t\right)^{1 / 2}+\frac{8 d T}{3} .
$$

By $\left(H_{6}\right)$ and Lemma 10 we have

$$
\begin{aligned}
& \left(\int_{0}^{T}\left|x(t)-x\left(t-\tau_{i}(t)-n_{i} T\right)\right|^{2} d t\right)^{1 / 2} \\
& \quad \leq \sqrt{2} \delta_{i}\left(\int_{0}^{T}\left|x^{\prime}(t)\right|^{2} d t\right)^{1 / 2}, \quad i=1,2 .
\end{aligned}
$$

Thus, it follows from (31), (32), (34), and (35) that

$$
\begin{aligned}
\gamma\left\|x^{\prime}\right\|_{L^{2}}^{2} \leq & \left(\sqrt{2} l_{1} \delta_{1}+\sqrt{2} l_{2} \delta_{2}+\frac{\left(b_{1}+b_{2}\right) T^{2}}{2 \pi^{2}}\right)\left\|x^{\prime}\right\|_{L^{2}}^{2} \\
& +\left[\frac{4 T^{3 / 2}}{3 \pi}\left[\left(a_{1}+a_{2}\right)+\left(b_{1}+b_{2}\right) d\right]+\|e\|_{L^{2}}\right]\left\|x^{\prime}\right\|_{L^{2}} \\
& +\frac{8 d T}{3}\left[\left(a_{1}+a_{2}\right)+\left(b_{1}+b_{2}\right) d\right] .
\end{aligned}
$$

Combining this with $\left(b_{1}+b_{2}\right) \gamma^{-1} T^{2}+2 \sqrt{2}\left(l_{1} \delta_{1}+\right.$ $\left.l_{2} \delta_{2}\right) \pi^{2} \gamma^{-1}<2 \pi^{2}$, we know that there exists $c_{1}$ such that $\left\|x^{\prime}\right\|_{L^{2}} \leq c_{1}$. Then

$$
\|x\|_{\infty} \leq d+\sqrt{T} c_{1} \triangleq M_{1} .
$$

Multiplying (19) with $x^{\prime \prime}(t)$ and integrating from 0 to $T$, then we have

$$
\begin{aligned}
& \left\|x^{\prime \prime}\right\|_{L^{2}}^{2} \\
& \leq \mid-\lambda \int_{0}^{T} g_{1}\left(t, x\left(t-\tau_{1}(t)\right)\right) x^{\prime \prime}(t) d t \\
& \quad-\lambda \int_{0}^{T} g_{2}\left(t, x\left(t-\tau_{2}(t)\right)\right) x^{\prime \prime}(t) d t \\
& \quad-(1-\lambda) m \int_{0}^{T} x(t) x^{\prime \prime}(t) d t \\
& \quad+\lambda \int_{0}^{T} e(t) x^{\prime \prime}(t) d t \mid \\
& \leq\left(g_{1 M_{1}}+g_{2 M_{1}}\right) \sqrt{T}\left\|x^{\prime \prime}\right\|_{L^{2}}+m M_{1} \sqrt{T}\left\|^{\prime \prime}\right\|_{L^{2}} \\
& \quad+\|e\|_{L^{2}}\left\|x^{\prime \prime}\right\|_{L^{2}},
\end{aligned}
$$

where

$$
\begin{aligned}
& g_{1 M_{1}}=\max _{t \in[0, T],\|x\|_{\infty} \leq M_{1}}\left|g_{1}(t, x(t))\right|, \\
& g_{2 M_{1}}=\max _{t \in[0, T],\|x\|_{\infty} \leq M_{1}}\left|g_{2}(t, x(t))\right| .
\end{aligned}
$$

Thus,

$$
\begin{aligned}
\left\|x^{\prime \prime}\right\|_{L^{2}} \leq & \left(g_{1 M_{1}}+g_{2 M_{1}}\right) \sqrt{T}+m M_{1} \sqrt{T} \\
& +\|e\|_{L^{2}} \triangleq M_{2} .
\end{aligned}
$$


Selecting $\eta \in[0, T]$ such that $x^{\prime}(\eta)=0$, then we have

$$
\left|x^{\prime}(t)\right| \leq \int_{0}^{T}\left|x^{\prime \prime}(t)\right| d t \leq \sqrt{T} M_{2} .
$$

Thus, from (35) and (41) we know that $\|x\| \leq M_{1}+$ $\sqrt{T} M_{2} \triangleq M$. It follows that

$$
\{x: x \in Y, x=\lambda A x, 0<\lambda<1\}
$$

is bounded. Therefore, by Lemma 8 , we obtain that $A$ has a fixed point $x^{*} \in \Omega$, where $\Omega=\{x: x \in Y,\|x\| \leq M\}$, and hence, it follows that (1) has a periodic solution.

\section{Proof of Theorem 3}

In this section, we will use Lemma 7 to prove Theorem 3.

Proof. Let $X, Y, A$ be defined and same as in Section 3. We prove that $A$ is Fréchet differentiable at 0 and

$$
\begin{aligned}
& \left(A^{\prime}(0) x\right)(t) \\
& \quad=K\left[r x^{\prime}(t)+\left(g_{1 x}^{\prime}(t, 0)+g_{2 x}^{\prime}(t, 0)\right) x(t)-m x(t)\right] .
\end{aligned}
$$

In fact, by $g_{1}(t, 0)+g_{2}(t, 0) \equiv e(t)$, we have $A 0=0$. Let

$(B x)(t)$

$$
=K\left[r x^{\prime}(t)+\left(g_{1 x}^{\prime}(t, 0)+g_{2 x}^{\prime}(t, 0)\right) x(t)-m x(t)\right] .
$$

We have

$$
\begin{aligned}
& \|A x-B x\| \\
& =\| K\left[f\left(x^{\prime}(t)\right)+g_{1}\left(t, x\left(t-\tau_{1}(t)\right)\right)\right. \\
& \left.+g_{2}\left(t, x\left(t-\tau_{2}(t)\right)\right)-m x(t)-e(t)\right] \\
& -K\left[r x^{\prime}(t)+\left(g_{1 x}^{\prime}(t, 0)+g_{2 x}^{\prime}(t, 0)\right) x(t)-m x(t)\right] \| \\
& \leq\|K\| \|\left[\frac{f\left(x^{\prime}(t)\right)}{x^{\prime}(t)}-r\right] x^{\prime}(t)+g_{1}\left(t, x\left(t-\tau_{1}(t)\right)\right) \\
& +g_{2}\left(t, x\left(t-\tau_{2}(t)\right)\right)-e(t) \\
& -\left(g_{1 x}^{\prime}(t, 0)+g_{2 x}^{\prime}(t, 0)\right) x(t) \| \\
& \leq\|K\| \|\left[\frac{f\left(x^{\prime}(t)\right)}{x^{\prime}(t)}-r\right]+g_{1 x}^{\prime}\left(t, \theta_{1} x\left(t-\tau_{1}(t)\right)\right) \\
& -g_{1 x}^{\prime}(t, 0)+g_{2 x}^{\prime}\left(t, \theta_{2} x\left(t-\tau_{2}(t)\right)\right) \\
& -g_{2 x}^{\prime}(t, 0)\|\| x \|
\end{aligned}
$$

where $\theta_{i}=\theta_{i}(t) \in(0,1), i=1,2$. Thus,

$$
\lim _{\|x\| \rightarrow 0} \frac{\|A x-A 0-B x\|}{\|x\|}=0,
$$

which implies that $A^{\prime}(0)$ exists and $A^{\prime}(0)=B$.

Now, we prove that 1 is not the eigenvalue of $A^{\prime}(0)$.

In fact, if there exists $x \in Y$ satisfying $A^{\prime}(0) x=x$, then $x$ is a solution of

$$
\begin{aligned}
-x^{\prime \prime}(t)-m x(t)= & r x^{\prime}(t)+\left(g_{1 x}^{\prime}(t, 0)+g_{2 x}^{\prime}(t, 0)\right) x(t) \\
& -m x(t) .
\end{aligned}
$$

Integrating (47) from 0 to $T$, we obtain

$$
\int_{0}^{T}\left(g_{1 x}^{\prime}(t, 0)+g_{2 x}^{\prime}(t, 0)\right) x(t) d t=0
$$

By $\left(H_{7}\right)$, there exists $t_{0} \in[0, T]$ such that $x\left(t_{0}\right)=0$.

Multiplying (47) with $x^{\prime}(t)$ and integrating it from 0 to $T$, we have

$$
\int_{0}^{T} r\left|x^{\prime}\right|^{2} d t=-\int_{0}^{T}\left(g_{1 x}^{\prime}(t, 0)+g_{2 x}^{\prime}(t, 0)\right) x(t) x^{\prime}(t) d t .
$$

By $\left(H_{7}\right)$ we have

$$
\int_{0}^{T} r\left|x^{\prime}\right|^{2} d t=0
$$

which implies that $x^{\prime}(t) \equiv 0$, and hence $x(t) \equiv 0$. It follows that 1 is not the eigenvalue of $A^{\prime}(0)$. By Lemma 7 we have

$$
i(A, 0)=i\left(A^{\prime}(0), 0\right)=(-1)^{\beta} .
$$

Next we prove that $\beta=1$. We can prove that

$$
\begin{aligned}
& -x^{\prime \prime}(t)-m x(t) \\
& =\lambda\left(r x^{\prime}(t)+\left(g_{1 x}^{\prime}(t, 0)+g_{2 x}^{\prime}(t, 0)\right) x(t)-m x(t)\right)
\end{aligned}
$$

has only one eigenvalue in $(0,1)$ and the algebraic multiplicity of it is 1 .

In fact, it is easy to see that $\lambda \in(0,1)$ is the eigenvalue of (52) if and only if $m+\lambda\left(g_{1 x}^{\prime}(t, 0)+g_{2 x}^{\prime}(t, 0)-m\right)=0$, that is,

$$
\lambda=\lambda_{0}=\frac{m}{m-g_{1 x}^{\prime}(t, 0)-g_{2 x}^{\prime}(t, 0)} \in(0,1) .
$$

In this case, (52) degenerates to

$$
x^{\prime \prime}(t)+\frac{m}{m-g_{1 x}^{\prime}(t, 0)-g_{2 x}^{\prime}(t, 0)} r x^{\prime}(t)=0
$$

It is not difficult to see that all the solutions of (54) are $x(t) \equiv$ const. Therefore the geometric multiplicity of $\lambda_{0}=$ $m /\left(m-g_{1 x}^{\prime}(t, 0)-g_{2 x}^{\prime}(t, 0)\right)$ is 1 . 
If $\left(I-\lambda_{0} A^{\prime}(0)\right)^{2} x(t)=0$, then $\left(I-\lambda_{0} A^{\prime}(0)\right) x(t)=c=$ const, and hence, $x(t)$ satisfies

$$
\begin{aligned}
& -x^{\prime \prime}(t)-m x(t) \\
& =\lambda_{0}\left(r x^{\prime}(t)+\left(g_{1 x}^{\prime}(t, 0)+g_{2 x}^{\prime}(t, 0)\right) x(t)-m x(t)\right)+m c,
\end{aligned}
$$

which implies that

$$
-x^{\prime \prime}(t)=\lambda_{0} r x^{\prime}(t)+m c .
$$

Integrating (56) from 0 to $T$, we obtain $c=0$, which implies that $x(t) \equiv$ const. It follows that the algebraic multiplicity of $\lambda_{0}=m /\left(m-g_{1 x}^{\prime}(t, 0)-g_{2 x}^{\prime}(t, 0)\right)$ is also 1 , and therefore, $\beta=1$. So $i(A, 0)=-1$. Letting $\delta>0$ small enough, then we have

$$
\operatorname{deg}\left(I-A, B_{\delta}, 0\right)=-1 .
$$

On the other hand, by the proof of Theorem 1, we know that there exists $M>0$ such that if $x \in Y, 0<\lambda<1$ satisfying $x=\lambda A x$, then $\|x\| \leq M$. Suppose $A x \neq x$ for $x \in Y,\|x\|=M$. By the homotopy invariance of topological degree, we have

$$
\operatorname{deg}\left(I-A, B_{M}, 0\right)=1,
$$

where $B_{M}=\{x \in Y:\|x\|<M\}$. It follows from (57) and (58) that

$$
\operatorname{deg}\left(I-A, B_{M} \backslash \bar{B}_{\delta}, 0\right)=2,
$$

which implies that $A$ has a fixed point $x$ in $B_{M} \backslash \bar{B}_{\delta}$ and $x$ is a nontrivial periodic solution of (1).

\section{Two Examples}

In this section, we give two examples to demonstrate the results obtained in previous sections.

Example 1. Consider the forced Rayleigh-type equation with period $2 \pi$ :

$$
\begin{gathered}
x^{\prime \prime}(t)+f\left(x^{\prime}(t)\right)+g_{1}\left(t, x\left(t-\tau_{1}(t)\right)\right) \\
+g_{2}\left(t, x\left(t-\tau_{2}(t)\right)\right)=e(t),
\end{gathered}
$$

where

$$
\begin{gathered}
f(x)= \begin{cases}e^{x}-1, & x \geq 0, \\
1-e^{-x}, & x \leq 0,\end{cases} \\
g_{1}(t, x)=\frac{1}{9} \sin ^{2} t x(t-\theta \cos t)+\cos t, \\
g_{2}(t, x)=\frac{1}{9} \cos ^{2} t x(t-\theta \sin t)+\sin t, \\
e(t)=\sin t, \quad \tau_{1}(t)=\theta \cos t, \\
\tau_{2}(t)=\theta \sin t, \quad \theta \in(0,1) .
\end{gathered}
$$

Conclusion. Equation (60) has at least one periodic solution with period $2 \pi$.

Proof. By (61) and (62), it is not difficult to see that the condition $\left(H_{1}\right)$ holds, $T=2 \pi$, and

$$
\begin{aligned}
& |f(0)|=0, \\
\left|g_{1 t}^{\prime}(t, x)\right| & =\left|\frac{1}{9} x \sin 2 t-\sin t\right| \\
\leq & \frac{1}{9}|x|+1, \quad \forall(t, x) \in R^{2}, \\
\left|g_{2 t}^{\prime}(t, x)\right| & =\left|-\frac{1}{9} x \sin 2 t-\cos t\right| \\
\leq & \frac{1}{9}|x|+1, \quad \forall(t, x) \in R^{2} .
\end{aligned}
$$

On the other hand, let $d=9, \gamma=1, \delta_{i}=\theta, l_{i}=1 / 9$, $b_{i}=1 / 9$, and $i=1,2$. If $\theta \in(0, \sqrt{2} / 2)$, then

$$
\begin{gathered}
x f(x) \geq|x|^{2}, \quad \forall x \in R, \\
\left(b_{1}+b_{2}\right) \gamma^{-1} T^{2}+2 \sqrt{2}\left(l_{1} \delta_{1}+l_{2} \delta_{2}\right) \pi^{2} \gamma^{-1}<2 \pi^{2} .
\end{gathered}
$$

Hence, $\left(H_{1}\right)-\left(H_{6}\right)$ are satisfied. Thus, by Theorem 1, (60) has at least one periodic solution with period $2 \pi$.

Example 2. If we replace $g_{1}(t, x), g_{2}(t, x)$, and $e(t)$ in Example 1 by

$$
\begin{aligned}
g_{1}\left(t, x\left(t-\tau_{1}(t)\right)\right)= & \frac{1}{9} \sin ^{2} t \cos \left(x\left(t-\tau_{1}(t)\right)+\frac{1}{2}\right) \\
& +\frac{1}{10} x\left(t-\tau_{1}(t)\right) \sin \frac{1}{2}, \quad \forall(t, x) \in R^{2}, \\
g_{2}\left(t, x\left(t-\tau_{2}(t)\right)\right)= & \frac{1}{9} \cos ^{2} t \cos \left(x\left(t-\tau_{2}(t)\right)+\frac{1}{2}\right) \\
& +\sin t, \quad \forall(t, x) \in R^{2},
\end{aligned}
$$

$e(t)=\sin t+(1 / 9) \cos (1 / 2)$, then we can obtain that $g_{1}(t, 0)+$ $g_{2}(t, 0) \equiv e(t)$, for all $t \in R$, and

$$
\begin{gathered}
g_{1 t}^{\prime}(t, x)=\frac{1}{9} \sin 2 t \cos \left(x+\frac{1}{2}\right), \\
g_{2 t}^{\prime}(t, x)=-\frac{1}{9} \sin 2 t \cos \left(x+\frac{1}{2}\right)+\cos t, \\
\left|g_{1 t}^{\prime}(t, x)\right| \leq \frac{1}{9}|x|+\frac{1}{18}, \\
\left|g_{2 t}^{\prime}(t, x)\right| \leq \frac{1}{9}|x|+\frac{37}{18}, \quad \forall(t, x) \in R^{2}, \\
g_{1 x}^{\prime}(t, x)=-\frac{1}{9} \sin ^{2} t \sin \left(x+\frac{1}{2}\right)+\frac{1}{10} \sin \frac{1}{2}, \\
g_{2 x}^{\prime}(t, x)=-\frac{1}{9} \cos ^{2} t \sin \left(x+\frac{1}{2}\right), \quad \forall t \in R, \\
g_{1 x}^{\prime}(t, 0)+g_{2 x}^{\prime}(t, 0)=-\frac{1}{9} \sin \frac{1}{2}+\frac{1}{10} \sin \frac{1}{2}<0
\end{gathered}
$$

$\forall t \in R$. 
Let $d=(10 /(9 \sin (1 / 2)))+((10 \sin (1 / 2)) /(9 \cos (1 / 2)))$, $\gamma=1, \delta_{i}=\theta, i=1,2, l_{1}=19 / 90, l_{2}=1 / 9, b_{1}=1 / 9$, and $b_{2}=1 / 9$. If $\theta \in(0,1)$; then

$$
\begin{gathered}
x f(x) \geq|x|^{2}, \quad \forall x \in R, \\
\left(b_{1}+b_{2}\right) \gamma^{-1} T^{2}+2 \sqrt{2}\left(l_{1} \delta_{1}+l_{2} \delta_{2}\right) \pi^{2} \gamma^{-1}<2 \pi^{2} .
\end{gathered}
$$

Hence, $\left(H_{1}\right)-\left(H_{8}\right)$ are satisfied. Thus, by Theorem 3, (60) has at least one nontrivial periodic solution with period $2 \pi$.

\section{Acknowledgments}

This work is sponsored by the project NSFC (11171032) and Beijing Excellent Training Grant (2010D005007000002).

\section{References}

[1] G. J. Ji, Z. X. Wang, and D. W. Lai, "Existence of periodic solutions to overvoltage models in electric power systems," Acta Mathematica Scientia A, vol. 16, no. 1, pp. 99-104, 1996 (Chinese).

[2] Z. Wang and D. Lai, "A delay differential equation appeared in the study of overvoltage," in Report to the Italian Symposium, vol. 12, 1984.

[3] C. H. Feng, "Existence and uniqueness of almost periodic solutions for some delayed differential equations appearing in a power system," Acta Mathematica Sinica, vol. 46, no. 5, pp. 931-936, 2003 (Chinese).

[4] T. A. Burton, Stability and Periodic Solutions of Ordinary and Functional-Differential Equations, vol. 178, Academic Press, Orlando, Fla, USA, 1985.

[5] J. Hale, Theory of Functional Differential Equations, Springer, New York, NY, USA, 1977.

[6] T. Yashizaw, "Asymptotic behavior of solutions of differential equations," in Differential Equation: Qualitative Theory (Szeged, 1984), vol. 47 of Colloquia mathematica Societatis János Bolyai, pp. 1141-1172, North-Holland, Amsterdam, 1987.

[7] Y. Kuang, Delay Differential Equations with Applications in Population Dynamics, Academic Press, New York, NY, USA, 1993.

[8] L. Peng, B. Liu, Q. Zhou, and L. Huang, "Periodic solutions for a kind of Rayleigh equation with two deviating arguments," Journal of the Franklin Institute, vol. 343, no. 7, pp. 676-687, 2006.

[9] C. Huang, Y. He, L. Huang, and W. Tan, "New results on the periodic solutions for a kind of Rayleigh equation with two deviating arguments," Mathematical and Computer Modelling, vol. 46, no. 5-6, pp. 604-611, 2007.

[10] B. Liu, "Existence and uniqueness of periodic solutions for a kind of Rayleigh equation with two deviating arguments," Computers \& Mathematics with Applications, vol. 55, no. 9, pp. 2108-2117, 2008.

[11] E. Zeidler, Nonlinear Functional Analysis and Its Applications. I: Fixed-Point Theorems, Springer, New York, NY, USA, 1986.

[12] D. Guo, Nonlinear Functional Analysis, Shandong Science and Technology Press, Ji Nan, China, 1985.

[13] S. Lu and W. Ge, "Periodic solutions for a kind of second order differential equations with multiple with deviating arguments," Applied Mathematics and Computation, vol. 146, pp. 195-209, 2003. 


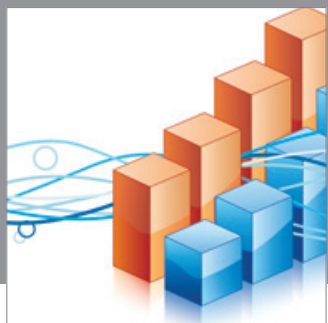

Advances in

Operations Research

mansans

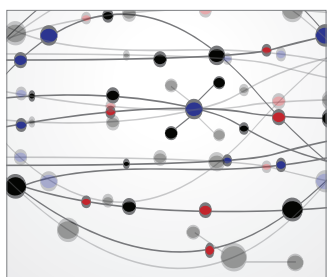

The Scientific World Journal
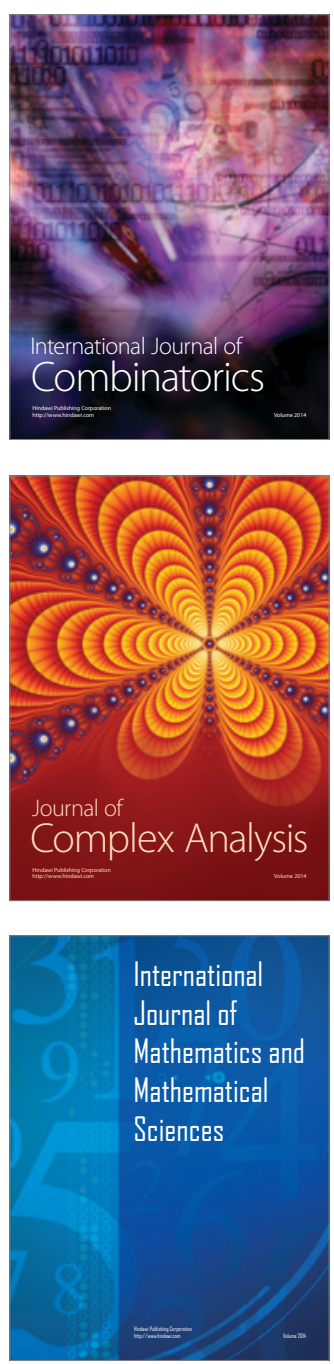
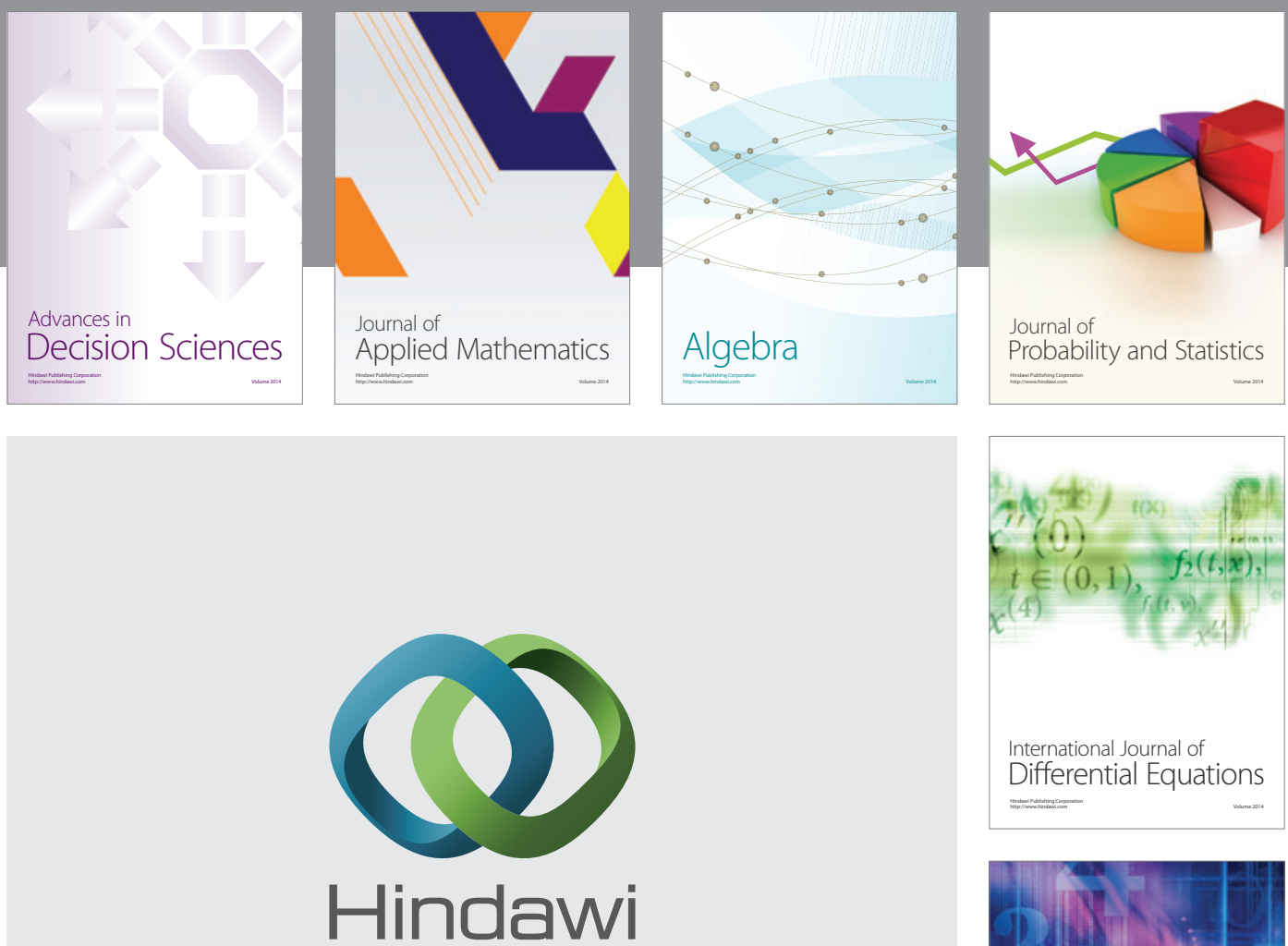

Submit your manuscripts at http://www.hindawi.com
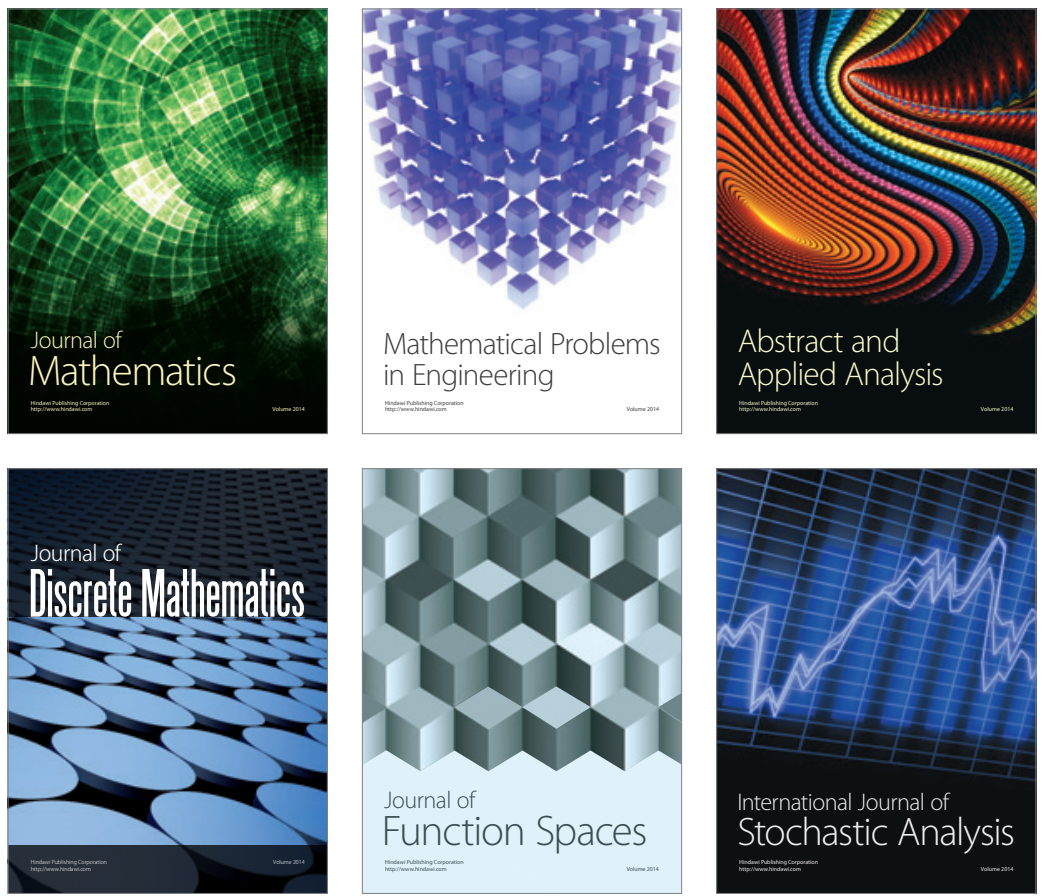

Journal of

Function Spaces

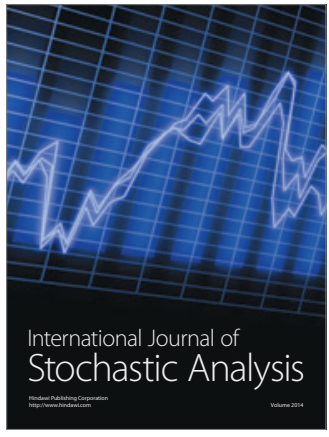

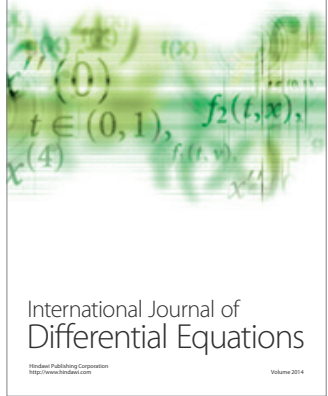
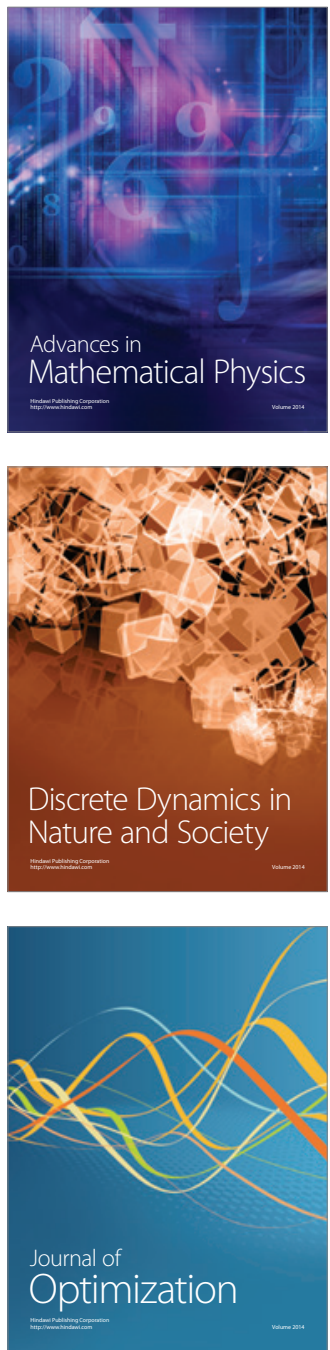